\title{
Contributions à la genèse du socialisme moderne : François Boissel, par le $\mathrm{D}^{\mathrm{r}}$ Carl Grünberg (Vienne)
}

\section{Jean-Numa Ducange}

Traducteur : Émilie Syssau

\section{(2) OpenEdition \\ Journals}

\section{Édition électronique}

URL : https://journals.openedition.org/ahrf/11892

DOI : 10.4000/ahrf.11892

ISSN : $1952-403 X$

\section{Éditeur :}

Armand Colin, Société des études robespierristes

\section{Édition imprimée}

Date de publication : 1 décembre 2010

ISBN : 978-2-200-92634-2

ISSN : 0003-4436

Référence électronique

Jean-Numa Ducange, "Contributions à la genèse du socialisme moderne : François Boissel, par le Dr Carl Grünberg (Vienne) », Annales historiques de la Révolution française [En ligne], 362 | octobredécembre 2010, mis en ligne le 25 mars 2011, consulté le 22 avril 2022. URL : http:// journals.openedition.org/ahrf/11892 ; DOI : https://doi.org/10.4000/ahrf.11892 


\section{Contributions à la genèse du socialisme moderne : François Boissel, par le $D^{\mathrm{r}}$ Carl Grünberg (Vienne) ${ }^{7}$}

Extraits sélectionnés par Jean-Numa Ducange.

Traduit de l'allemand par Émilie Syssau.

[...] Les pages suivantes cherchent à améliorer la connaissance d'un écrivain socialiste du $\mathrm{XVIII}^{\mathrm{e}}$ siècle jusqu'à présent complètement ignoré, et ainsi à éclairer la genèse du socialisme.

[...] L'ouvrage dans lequel Boissel a principalement exprimé ses idées au sujet d'une réorganisation sociale est le Catéchisme. Les écrits postérieurs - plus particulièrement les Entretiens - ne font la plupart du temps que répéter ce même raisonnement, et souvent même citent mot pour mot le Catéchisme.

[...] Si l'on fait abstraction d'un contenu souvent absurde, tous les écrits de Boissel sont rédigés dans un style des plus emphatiques et il en coûte quelques efforts pour se retrouver dans les phrases qui reviennent sans cesse et pour y deviner les pensées de l'auteur. L'exposé qui suit montrera dans quelle mesure j'y suis parvenu.

L'observation du monde extérieur dans son ensemble et de la position qu'y occupe l'homme nous enseigne - expose Boissel - que celui-ci est doté exactement des mêmes besoins, du même désir de vivre et des mêmes fonctions indispensables que tous les autres êtres vivants. Mais alors que la nature et son créateur, Dieu, ont donné à ces derniers l'instinct qui les conduit sûrement et infailliblement selon des règles immuables, ils l'ont refusé à l'homme. Celui-ci possède à la place de l'instinct la raison, «cette faculté de [l']âme, qui nous rend susceptibles de connoissances et de les tourner à notre plus grand avantage $»^{8}$. Elle constitue le caractère distinctif essentiel entre lui et le monde animal. Elle le rend également capable de se créer lui-même, en marge de l'ordre vital naturel, un autre ordre, étranger et fermé aux autres êtres vivants : la société morale (l'ordre moral ou social). On le voit, Boissel identifie la notion de société à celle de société reposant sur des bases morales.

Cet ordre vital moral doit être raisonnable; ce n'est que lorsqu'il est raisonnable qu'il est en retour moral. Il s'agit donc tout d'abord de

(7) Carl Grunberg, «Einige Beiträge zur Entwicklungsgeschichte des modernen Sozialismus : François BoIssEL », dans Zeitschrift für Staatswissenschaft, 1891, cahier II, p. 207-258. Traduction française : Émilie Syssau, traductrice diplômée, juin 2008. père Gérard.

(8) Borssel, Catéchisme du Genre Humain, p. 120 et passim, ainsi que Les Entretiens du 
reconnaître les vrais principes raisonnables et moraux qui doivent être déterminants lors de la formation de la société. Alors seulement il sera possible d'examiner la teneur morale de l'ordre social existant, alors seulement on sera en mesure de l'améliorer et de le réorganiser de façon appropriée, si et dans la mesure où il n'apparaît pas conforme à la raison.

La raison humaine manque cependant, contrairement à l'instinct, de règles solides et immuables. S'y ajoute le fait qu'une évolution millénaire nous a précédés. Non seulement la raison n'y est pas restée insensible, mais elle semble au contraire en être le résultat. "Résultat intellectuel de l'organisation, de l'éducation, des sensations, des inclinations, \& des habitudes des hommes, qui varient selon les climats, chez les différents peuples \& même dans chaque individu », elle s'exprime de manière aussi variée que les éléments qui influencent son évolution. Les opinions des hommes, c'est-à-dire " les jugements ou les résultats des délibérations sur les objets que la raison soumet à son examen » (Cat. 118) n'en sont pas moins divergents.

Il ne peut cependant y avoir qu'une seule vérité. Il ne peut exister plusieurs opinions également justes et également raisonnables à propos d'un même objet. Comment donc reconnaître cette vérité unique?

Nous devons à ces fins nous en tenir à la nature elle-même, « de qui nous tenons notre existence physique, ainsi que tous les moyens de la conserver » (Cat. 3 et passim). Que nous enseigne alors la nature?

Notre auteur répond à cette question de la façon suivante : tous les hommes ont des besoins et le désir de les satisfaire aussi longtemps et aussi parfaitement que possible. Ils sont eux aussi soumis aux « lois prescrites à tous les êtres vivants : se soutenir, se conserver, se reproduire \& se perpétuer les uns par les autres » (Cat. 121) ${ }^{9}$. Ce qui circonscrit également la série des besoins de l'homme et en donne la classification.

Le but du genre humain, poursuit Boissel, est, à l'instar de celui du monde animal, d'être heureux. «Le bonheur consiste dans la santé, la force \& l'adresse de son corps, dans la paix \& le contentement de son âme, $\&$ dans le nécessaire pour la vie la plus frugale, c'est-à-dire pour une satisfaction des besoins dans leurs véritables limites » $\left(\right.$ Cat. 82) ${ }^{10}$.

(9) NdT : les citations du Catéchisme du genre humain en italiques sont données en français par C. Grünberg, lorsqu'elles étaient traduites en allemand dans son article, elles ont été rétablies en s'appuyant sur la deuxième édition de l'ouvrage (1792) dans le respect de la graphie originale.

(10) Boissel précise l'expression « frugal»: «Parce que la frugalité n'exprime que le vrai terme des besoins et que par de là on ne peut concevoir que d'excès préjudiciables à ce même bonheur ». 
Malgré l'inégalité naturelle des hommes concernant leurs dispositions physiques et intellectuelles, et malgré la diversité des besoins qui en résulte, on ne peut cependant pas dire que quiconque soit davantage favorisé dans ses pulsions pour les satisfaire. Personne n'a davantage de légitimité qu'un autre à s'imposer. C'est-à-dire que, dans sa prétention à satisfaire ses besoins, aucun individu humain n'est différent de l'autre; ils sont donc tous égaux.

La nature elle-même et son auteur, Dieu, ne s'occupent toutefois avant tout que du maintien de l'espèce, et non de l'individu. Dans l'état naturel, chacun s'impose ainsi comme il le peut - les moyens lui en sont donnés. Reste cependant à savoir si cela doit se produire contre les autres et à leur préjudice, ou si ce but ne peut pas être mieux atteint en se fondant sur l'harmonie de tous. Nous verrons bientôt comment Boissel répond à cette question. Poursuivons d'abord son raisonnement.

Pour améliorer la lutte pour l'existence - poursuit-il - la nature a fait de l'homme une créature sociale. Le désir de se sociabiliser lui est inné et trouve sa première expression dans les rapports entre la mère et ses enfants (Cat. 154 sq. et passim). Puisque l'homme, naissant sous la dépendance de la nourrice, qu'on appelle sa mère, \& ne pouvant exister alors que par ses tendres soins, devient naturellement l'associé de sa mère ainsi que de ses frères \& sœurs » (Cat. 115-116). Ainsi émergent de petits groupes d'individus et d'intérêts qui fusionnent en de plus grands pour finir par s'unir en peuples et en nations. Mais tous partagent les mêmes fondements et le même objectif, à savoir celui de faciliter la lutte pour l'existence contre les forces naturelles, éventuellement avec d'autres groupes et individus (Cat. 116).

Il en va ainsi de l'ordre naturel (la société dans l'ordre physique). Selon Boissel, toute lutte entre les individus ou entre les communautés est en soi absolument illégitime et inutile. Elle ne le deviendrait qu'en cas de nécessité, « si la terre n'avait pas de quoi nourrir ses habitants ». Une condition qui ne serait cependant pas exacte (Cat. 8).

Néanmoins, il existe également, poursuit-il, d'autres origines à la lutte mutuelle des hommes. Les individus n'apportent en venant au monde rien «qu'une perfectibilité à cultiver, un égoïsme sans borne à dompter \& des passions qui, selon qu'elles sont bien ou mal dirigées, font le bonheur ou le malheur de notre vie » (Cat. 3). La nature et la volonté impénétrable de Dieu confient ainsi aux hommes le soin de concevoir leur destin, les dotant à part égale des moyens de détruire comme de créer leur bonheur, lequel dépend dans les deux cas de leur bon vouloir. C'est ce qui les distingue précisément du reste du monde animal, et la conscience de 
ce phénomène exclut tout ressassement à se demander s'il n'aurait pas été préférable que la nature ne mette pas ce pouvoir entre nos mains (surtout Cat. 171 et passim).

Que les hommes choisissent l'égoïsme pour moteur de leur vie et de leurs actes, et le plus fort atteindra simplement le sommet et y restera : non parce qu'il en a le droit, mais pour l'unique raison qu'il est plus fort.

Et les conséquences? « S'il a un goût dominant pour les femmes, il s'emparera de toutes celles qui lui feront plaisir; s'il est animé du désir de prédominer \& de se faire valoir, il forcera ses semblables à le suivre $\&$ à lui obéir. Il s'emparera du fruit des travaux des plus laborieux \& des plus paisibles. Il suivra l'exemple des lions \& des tigres. Les plus faibles lui cèderont $\&$ lui obéiront pendant tout le temps qu'il sera assez fort pour se faire craindre. Après lequel temps, celui qui se sentira le plus fort $\&$ qui n'aura de meilleur conseil que le même égoïsme \& les mêmes inclinations, en usera selon l'exemple qu'il en aura reçu \& appris » (Cat. 160).

Mais de cette manière, nul n'est besoin de le démontrer, les uns rendront les autres malheureux sans être eux-mêmes plus heureux. Dieu et la nature se préoccuperont peu de ce que «les hommes se dégradent, s'oppriment et se détruisent les uns par les autres : l'espèce ne se perdra pas plus à l'avenir que par le passé » (Cat. 171), et c'est pour eux l'essentiel.

L'égoïsme ne doit donc pas être érigé en fondement de la vie du genre humain. Celui-ci doit plutôt suivre des mobiles altruistes. Eux seuls garantissent à tous le plus grand bonheur possible. Ils sont donc les seuls raisonnables et donc aussi les seuls à suivre la morale. Cet état de fait se révèle à notre auteur par l'observation suivante.

Nous avons précédemment souligné qu'il reconnaît l'inégalité naturelle des hommes sous le rapport de leurs dispositions physiques et intellectuelles, et par conséquent également l'inégalité de leurs besoins, et nous avons vu comment il parvient tout de même à la notion d'égalité de tous. Il suppose en effet que tous ont le même droit à l'existence ${ }^{11}$ et il est un des représentants les plus résolus de ce principe.

«L'homme, explique Boissel, arrive nu sur la terre, comme il y retourne de même après sa mort (Cat. 59-60). Comme il n'apporte rien, on ne peut pas prétendre qu'il ait un droit à quoi que ce soit. La notion de «droit » comme pouvoir revenant à une personne à l'exclusion de toute

(11) Concernant la notion de droit à l'existence et ses représentants d'alors, cf. en particulier Anton Menger, Das Recht auf den vollen Arbeitsertrag [Le droit au plein rendement du travail], Stuttgart, 1886, p. 8-10. 
autre est du reste étrangère à la nature. Elle ne voit le jour que par et dans la société. Mais là encore, non comme une nécessité conceptuelle, mais en réaction au fait que les individus s'arrogent de tels pouvoirs exclusifs. Et une telle prétention ne peut bien évidemment pas justifier un droit réservé à ces seuls derniers, mais fait valoir pour tous les autres en même temps le même droit au même objet et avec le même contenu. "Nul ne peut s'arroger dans ce monde ni établir un droit en sa faveur au préjudice de son semblable, sans établir en même temps en faveur de son semblable le même droit contre lui » (Cat. 62). En d'autres termes : comme dans la nature personne n'est plus légitime qu'un autre, tous ont les mêmes droits. Ils ont donc tous le même droit aux objets appropriés et nécessaires à la satisfaction de leurs besoins, chacun en fonction de ses besoins, mais sur la base seulement du travail. Nous y reviendrons en détail un peu plus loin.

La réflexion la plus élémentaire, estime Boissel, devrait enseigner à chacun que la meilleure garantie de son droit à l'existence passe non seulement par le non-refus de ce même droit à l'Autre, mais encore et surtout par sa reconnaissance et l'effort déployé pour le faire accepter. Ce ne serait qu'ainsi qu'il échapperait au revers inévitable s'opposant à l'affirmation exclusive de sa personnalité. Ce ne serait qu'en abandonnant la lutte contre tous qu'il empêcherait également la lutte de tous contre lui. C'est ainsi cependant que l'on parviendrait à chercher et à trouver son propre bonheur dans celui de la collectivité (Cat. et Les Entretiens, passim).

Ceci étant dit, argumente ensuite Boissel, ni le but de la vie humaine, ni le moyen d'y parvenir ne prêtent à confusion. Si les hommes s'attachent maintenant à créer l'« ordre social ou moral », ils ne doivent de toute évidence s'appuyer que sur les fondements qui sont ceux de la société « dans l'ordre physique ». Seulement voilà, il est impératif que les deux objectifs relevant de la même conception, à savoir faciliter la lutte pour l'existence et garantir cette dernière, soient désormais réalisés dans la quasi-totalité de la masse. Car c'est là seulement que réside la finalité de l'ordre social. C'est son unique droit à l'existence. Les hommes ne préféreraient-ils pas sinon l'état naturel? Et comme on a constaté que ces objectifs ne pouvaient être atteints que si l'égalité et le droit égal de tous à l'existence étaient reconnus et mis en œuvre, toutes les forces doivent être dirigées vers ce but dans l'ordre social délibérément créé par l'homme et ne doivent en rien contredire son organisation. Sinon, l'ordre social porte son nom à tort : il est alors déraisonnable et immoral. Le grand progrès de l'ordre social - tel qu'il doit être - sur le règne de la nature réside dans le fait que, fort de la reconnaissance de l'égalité et de l'égalité de droit de tous à exister, il doit être également pensé en termes de maintien équitable 
de tous les individus alors que dans la nature il est avant tout question du seul maintien de l'espèce (Cat. 4, 59 et passim).

Formé d'après le modèle de la société naturelle la plus primitive, à savoir les relations entre la mère et ses enfants, l'ordre social doit être à ses membres ce que la mère est à ses enfants dans l'état naturel. "Dans l'ordre moral ou social, nous appartenons tous à la société de qui nous tenons notre existence morale ou sociale. Nous vivons aussi sous sa dépendance; c'est donc à la société, mère de tous les membres qui la composent, d'élever ses enfants. Il faut donc qu'elle soit organisée de façon que les enfants trouvent dans son sein les mêmes ressources pour les besoins physiques que celles qu'ils ont trouvées en naissant dans le sein de leur nourrice ou mère naturelle» (Cat. 4). La "morale \& toutes les institutions humaines ne peuvent donc avoir d'autre objet, ni d'intérêt plus réel, que de prévenir les causes physiques \& morales de la douleur \& de rapprocher toutes celles du plaisir » (Cat. 39-42, 168 sq). À l'inverse, « toutes les institutions sociales, soit qu'on les appelle humaines, soit divines, qui préjudicient à l'existence, aux fonctions \& besoins naturels de l'homme social sont contre les droits de la nature \& par conséquent de son auteur ", c'est-à-dire immorales et déraisonnables (Cat. 7).

Chaque membre de la société est donc, d'après notre auteur, en droit de fixer à la société les exigences développées à l'instant. Cette légitimé découle de l'être des choses. Nul n'est besoin d'un contrat social dans le sens de Rousseau. Boissel reconnaît en effet qu'il n'en a jamais existé de tel historiquement. « Mais, ajoute-t-il, ce contrat existe, puisque la nature \& son auteur en ont imprimé les clauses \& les conditions dans le cœur de tous les hommes sensibles \& pensans » (Cat. 90).

Une fois parvenu, en s'appuyant sur des considérations générales, aux résultats qui viennent d'être présentés, Boissel enchaîne par l'examen de l'ordre effectif des choses en lui appliquant les normes acquises. Comme nous connaissons ses critères, nous n'avons pas à nous étonner du résultat de sa critique, et à peine à souligner sa radicalité.

Il existe essentiellement trois institutions qui, dit-il, impriment leur marque à l'ordre social actuel; la religion, le mariage et la propriété ou, si l'on veut, cette dernière seule, car la trinité complète des institutions sociales fondamentales se ramène à la notion de propriété. Boissel définit en effet la religion comme la propriété de Dieu et des choses divines, et le mariage comme la propriété des femmes.

Comme toutes les institutions sociales, poursuit-il dans son argumentation, on ne peut considérer la propriété, la religion et le mariage que dans la mesure où « par l'expérience qu'on en a faite, ces institutions 
sacrées ont servi \& servent encore aujourd'hui au bonheur des hommes » (Cat. 6). L'expérience séculaire prouverait cependant le contraire, et que la responsabilité de tous les maux indicibles dont souffre l'humanité incomberait justement à ces trois clefs de voûte de l'ordre social actuel (ibid. et passim). Qu'est-ce en effet que la propriété? Les juristes la définissent comme «la liberté de disposer de ce qu'on dit nous appartenir, comme bon nous semble » (Cat. 92). Une piètre et terne définition qui ne satisfait pas notre auteur. Il en donne pour sa part une autre : « la propriété $[\ldots]$ est $[\ldots]$ le privilège exclusif de jouir [des terres], \& par conséquent de bannir du globe terrestre les races futures; de faire mourir de faim, de soif ou de froid celui qui n'a pas de propriété, si mieux n'aime ce dernier devenir l'esclave du propriétaire, \& ce dernier l'agréer à cette condition; sinon, pendu, comme voleur, ou empalé ou rompu vif, comme assassin » (Cat. 93). ${ }^{12}$

La propriété, poursuit Boissel, contredit donc le droit de tous à l'existence. Elle n'est d'ailleurs pas un droit, mais simplement une réalité acquise par la violence, et qui ne peut de même être conservée que par la violence. En fait, elle ne repose sur aucun droit divin ou humain, comme on l'affirme si souvent - car elle s'oppose aux deux de manière implacable - mais seulement sur le désir insatiable, l'orgueil, la violence et la duplicité des hommes. Ainsi créée par eux, elle convoque à son tour et nourrit sans cesse " l'intérêt désastreux de ne rapporter qu'à soimême tout ce qui ne doit être rapporté qu'à la masse générale pour être distribué selon les besoins de chaque membre de la société » (Cat. 96 et passim).

L'institution de la propriété privée aurait encore suscité les échanges et le commerce. Non pas des échanges dans le sens d'un véritable ordre social, c'est-à-dire « la communication des moyens de se rendre \& de se conserver heureux les uns par les autres ». Il faudrait plutôt entendre par là la somme des relations qui naissent entre les individus, les familles et les peuples à travers les besoins et les inclinations créées par l'ordre dominant des choses, et dont les hommes sont maintenant les misérables jouets (Cat. 126). Le commerce et les échanges ne donnent donc pas un avantage à tout le monde, mais seulement à une petite minorité avisée et habile, dont l'enrichissement s'accompagne de la tromperie et de l'appauvrissement de la grande majorité (Cat. 110 sq.).

(12) Ici, comme presque toujours, même s'il consent des exceptions, Boissel parle de la propriété du sol et des biens fonciers (propriété de terres, partage de terres), sans cependant considérer la propriété d'un bien mobilier comme acceptable ou ne serait-ce que moins condamnable. 
Pour les besoins du commerce et des échanges tels que les connaît l'ordre social actuel, on aurait créé le numéraire : « des espèces d'or \& d'argent auxquelles ils ont attaché tant de valeurs qu'on peut tout acheter avec ces espèces » (Cat. 92). Le numéraire serait aussi superflu dans un ordre social véritablement raisonnable qu'il est indispensable dans l'actuel. Naturellement, il ne servirait, comme ces dernières d'ailleurs, après tout, qu'à accroître la corruption générale (Cat. 110-111) ${ }^{13}$.

La propriété privée poserait ainsi les bases de la misère absolue parmi les hommes. Elle encouragerait partout et toujours la privation de liberté, l'avilissement, la dépendance des hommes les uns par rapport aux autres et vis-à-vis de leurs passions. Ceci vaudrait non seulement pour les individus au sein d'un groupe mais aussi pour les relations de ces derniers entre eux. Procédant du «bellum omnium contra omnes » [combat de tous contre tous. $\mathrm{NdT}$ ], elle le perpétue. Et, de la même façon qu'entre les individus, seuls le poing et la violence brutale seraient en vigueur entre les peuples et les États.

Naturellement, Boissel ne laisse pas passer l'occasion de se déclarer contre la guerre. « Nous appelons cet art monstrueux de nous entr'égorger, un droit, une institution humaine \& même divine, quoiqu'il n'en peut exister dont les effets soient plus humains \& plus diaboliques » (Cat. 8). Et il n'aurait certainement pas moins décrié notre époque marquée par des budgets militaires exorbitants et la succession incessante d'inventions guerrières. «C'est sur cet art abominable, sur cette institution exécrable, la honte comme le malheur du genre humain, que les États les plus éclairés de l'Europe fondent encore aujourd'hui leur salut, leurs espérances, leur héroïsme, leur gloire, leur prééminence, leur destinée, en un mot, toutes leurs brillantes chimères » (Cat. 9). Celui qui fabriquerait les meilleurs instruments criminels bénéficierait des plus grands honneurs. Et celui qui parviendrait à inventer un instrument criminel permettant de réduire en un instant une armée entière serait assurément célébré et honoré comme étant le génie par excellence (Cat. 109-110).

Boissel critique la propriété - comme auparavant, d'après son principe selon lequel seul le travail donne le droit à l'existence, qui découle de la définition du droit de propriété précédemment introduite - non seule-

(13) Remarquons par ailleurs que Boissel n'a aucune idée de la nature du numéraire et qu'il est partisan de l'idée selon laquelle les hommes pourraient arbitrairement prêter à tout objet de leur choix le caractère de monnaie (les hommes ont été les maîtres de convenir de toute autre espèce de matière). «Qu'on ait précisément choisi les métaux nobles s'expliquerait par le fait qu'il est entré dans le plan des imposteurs, qui s'étoient emparé du pouvoir céleste » (Cat. 116 sq.). 
ment comme l'origine intrinsèque de tous les maux de la société mais aussi comme la source d'un revenu sans travail (passim).

Et pour résumer, il proclame plein de rage : «J'avoue que, sous des rapports aussi monstrueux, mon esprit ni mon cœur ne sauroient voir, dans un propriétaire, au lieu d'un homme social, qu'un monstre antisocial » (Cat. 68).

Mais les hommes - poursuit-il - ne se sont pas contentés de s'arroger des droits exclusifs sur les objets qui leurs sont extérieurs; ils les ont même revendiqués comme étant leurs. Cette violence trouve son expression dans les institutions de l'esclavage et du mariage.

Les objections de Boissel contre l'esclavage peuvent être ici passées sous silence (cf. Cat. 139 sq.).

Ses développements sur, ou plutôt contre le mariage et toutes ses conséquences, méritent au contraire l'attention, parce qu'ils nous montrent parfaitement la hardiesse avec laquelle il a abordé son problème et a mené à terme la réflexion sur ses hypothèses.

Le mariage, c'est-à-dire « les règles [et] les cérémonies [...] que les hommes ont inventées, dans les différents climats, pour l'union des deux sexes » (Cat. 99), est pour lui « un attentat des plus formels à la liberté, surtout de la femme, un divorce avec le reste du genre humain, une ligue redoutable contre ses semblables " (Cat. 101). Il lui apparaît donc non seulement comme quelque chose d'immoral en soi - je reviens immédiatement à ce point - mais aussi comme ayant par ailleurs des conséquences extrêmement pernicieuses, parce qu'il est synonyme de consolidation et de perpétuation de la propriété privée et de l'ordre économique, juridique et social fondé sur celle-ci.

La propriété privée est très certainement, poursuit-il, une conséquence de l'égoïsme humain; et, de sa conséquence, elle en redevient la cause. L'égoïsme ne se manifeste cependant pas de façon aussi forte et aussi explosive chez tous les hommes - et même, chez beaucoup, ne se manifeste pas du tout. Les répercussions pernicieuses du droit de propriété ne sont ainsi conceptuellement pas moindres, mais s'affirment encore davantage, lorsque la famille accède à la propriété privée. La famille, c'est-à dire la paternité, laquelle serait impensable sans le mariage. Elle est « devenue un titre \& la cause d'un égoïsme sans borne [...] fondé sur le penchant naturel d'un père pour ses enfants $\&$ sa famille, de sacrifier l'univers, si faire ce pouvait, $[\ldots]$ pour le transmettre à ses enfants $\&$ à sa postérité la plus reculée » (Cat. 100).

Bien que notre auteur manque de force, de clarté et de précision dans son expression, on voit cependant clairement qu'il s'en prend au 
mariage, c'est-à-dire à la famille, parce qu'il a pour conséquence le droit successoral, et donc l'immortalité de la propriété privée. [...]

La façon singulière dont Boissel répond à la question de la légitimité de la société, qui intervient de façon à réguler les relations entre homme et femme, est de fait des plus intéressantes et ne peut être contournée.

Boissel considère la satisfaction de l'instinct sexuel comme le plus important des plaisirs (Cat. 165). La raison de ce phénomène pourrait s'expliquer par le fait que « le but principal de l'auteur de tous les êtres a été d'en conserver \& d'en perpétuer chaque espèce ». Ce n'est que pour cela, et non pour assurer une source de plaisirs à l'homme, que la nature aurait créé le penchant des sexes l'un pour l'autre et la volupté que l'on trouve dans la satisfaction de l'instinct sexuel. Ceci serait d'autant plus évident si l'on considère que les hommes peuvent non seulement vivre sans union sexuelle, mais aussi, même, que survenant à l'excès, le plaisir affaiblirait l'individu et finalement le détruirait. Il remplirait précisément une fonction purement animale et obéirait ainsi à une force indépendante de sa volonté, voire agirait dans l'ensemble contre celle-là même.

Les hommes s'unissent-ils de leur côté avec la volonté de conserver l'espèce ? Certainement pas, mais plutôt parce qu'ils en conçoivent du plaisir! Que signifie donc le mariage, c'est-à-dire la régulation sociale des relations sexuelles? La société veut-elle ainsi venir en aide à la nature, et devons-nous donc entrevoir dans le mariage une intervention délibérée de la société visant le maintien de l'espèce? Personne n'en conviendrait. Il serait d'ailleurs ridicule, et de toute façon inutile, de vouloir ainsi venir en aide à la nature. La nature veillerait à ce que l'espèce humaine ne s'éteigne pas. La société ne devrait s'occuper que d'entretenir ses membres actuels, et ainsi ne restreindre leur libre activité que dans la mesure où un inconvénient en résulterait pour les autres. On ne pourrait cependant parler ainsi de la satisfaction de l'instinct sexuel en général.

Le seul ascendant de la société dans ce domaine ne pourrait être - dans l'intérêt des individus - que de leur enseigner les conséquences néfastes d'une satisfaction démesurée de l'instinct sexuel et de la leur déconseiller (Cat. 43 sq., 100 sq.).

Le mariage serait donc, conclut Boissel, dans tous les cas une institution superflue et inutile. Mais pas seulement. Il serait également tout simplement dangereux, parce qu'il restreindrait la liberté, notamment de la femme. $[\ldots]$

La religion vient encore s'ajouter à la famille pour donner un autre appui à l'ordre du droit privé - poursuit Boissel dans sa critique de ce dernier. 
Il définit la religion comme « les moyens qu'ont employés \& les institutions qu'ont établies originairement les esprits forts pour commander aux esprits faibles au nom de la divinité qu'ils ont fabriquée $\&$ fait parler comme ils ont voulu » (Cat. 103). [...]

Je le souligne une fois encore : les attaques de Boissel ne se dirigent nullement contre l'existence d'une créature divine, mais seulement contre le Dieu des Églises et des prêtres. Il croit à une cause première de toute chose. Cette idée, soit l'idée même d'un Dieu, pense-t-il, ne serait cependant pas innée aux hommes, mais le résultat nécessaire de leurs premières expériences, ainsi que de la réflexion à leur sujet. Car dès que l'homme commencerait à penser, il devrait reconnaître qu'il s'est aussi peu créé luimême que les nombreux autres objets qu'il perçoit (Cat. 188 et passim).

Cette constatation et le sentiment de dépendance à Dieu et à la nature, qui en découle, apparaît même à notre auteur comme une condition sine qua non à un ordre social véritablement moral. En effet, de la conscience de ce que tout notre être, ainsi que toutes nos actions, devraient prendre leur véritable origine et leur fin en Dieu, en lien avec la conception de la supériorité de la divinité, découlerait la conclusion impérative que l'ordre social devrait être véritablement moral, et d'autre part que l'ordre dominant des choses ne pourrait avoir Dieu pour auteur - comme on l'affirme - mais serait plutôt contre nature et hostile à Dieu (Cat. 189 sq.). Si ceux qui s'intéressent à la perpétuation de cet ordre se réclamaient tout de même de la divinité, cela prouverait seulement que cette dernière, telle qu'on nous la représente, ne serait qu'un épouvantail permettant de servir des buts égoïstes (passim).

Il est impossible - poursuit Boissel - de discerner l'origine et la nature des choses. Ce n'est d'ailleurs aucunement une condition du bonheur humain. Les hommes pourraient manquer de discernement tout aussi bien dans l'état naturel que dans l'ordre social, sans enfreindre aucun des véritables commandements moraux et de ce fait sans détériorer les conditions de leur bonheur. La parfaite indifférence de la divinité, cette créature infiniment supérieure, à ce que les vermisseaux humains la reconnaissent et la vénèrent, ne la vénèrent pas, ou même l'insultent, irait de soi si l'on comparait le caractère fini de l'homme avec l'éternité de la créature divine. Que nous soyons cependant dominés, pense-t-il, par la curiosité et la quête d'approfondir les choses, que notre âme soit toujours remplie du souhait d'un bonheur supérieur au bonheur terrestre : ceci pourrait bien être considéré comme « le fondement d'une croyance \& d'une espérance [...] d'une autre destinée $\&$ d'une autre vie où cette curiosité vaine, mais naturelle, où ces désirs impuissants, mais naturels, seront pleinement satis- 
faits 》 (Cat. 20 sq., 204 sq.). Jamais cependant n'en découlerait la nécessité des religions, d'un culte, d'une vénération divine organisée. Jamais ceci ne pourrait servir à légitimer l'intolérance, la terrible autodestruction du genre humain et la misère indicible que les religions ont entraînées et entraînent dans leur sillage (passim). [...]

La propriété, la famille (le mariage) et la religion sont les fondements de notre structure sociale! proclame furieusement Boissel. Et elle s'y conforme! Elle mérite bien d'être qualifiée d'homicide, de mercenaire et d'antisociale. « J'appelle [l'ordre social actuel] mercenaire parce qu'il n'invite à faire le bien, que par l'espoir d'une récompense, \& à éviter le mal, que par la crainte d'un châtiment [...]. Je l'appelle homicide, parce qu'il arme le fils contre le père, le frère contre le frère, les familles contre les familles \& les peuples contre les peuples pour [...] nous faire détruire les uns par les autres. Je l'appelle antisocial, parce qu'il engendre l'intérêt désastreux de ne rapporter qu'à soi, ce qui ne doit être rapporté qu'à la masse générale de la société pour être distribué, selon les besoins de chacun de ses membres » (Cat. 89).

Si les soubassements de la société sont mauvais, il en va aussi naturellement de même des institutions conçues pour la protéger. On parle en effet par trop, estime Boissel, de la loi, du droit et de la justice. Dans les faits cependant, les « lois » sont les obligations que les forts imposent aux faibles pour maintenir le désastreux ordre actuel des choses et en profiter le plus possible au détriment du plus grand nombre; la «justice» est l'exercice constant de la volonté des forts visant le maintien de ces relations; le «droit» est certes défini comme ars aequi et boni, iusti atque iniusti notitia [l'art de l'équité et du bien, la connaissance du juste et de l'injuste. NdT], mais cette définition serait globalement incorrecte et ambiguë si l'on considérait ce que signifient réellement la loi et la justice (Cat. 118-119).

Avec les progrès de la civilisation, on aurait toutefois, et cela à nouveau essentiellement dans l'intérêt des gouvernants qui refusaient de se voir menacés, voire même oppressés, par le règne démesuré de l'égoïsme, cherché à le maîtriser. Un système complet de mesures et d'institutions préventives et répressives serait ainsi né. « Mais ce que nous appelons droit civil et politique n'a offert jusqu'à présent, et ne continuera à offrir, qu'une mauvaise aide, car les causes de l'égoïsme se perpétuent et ne cessent d'agir » (Cat. 173, 68 sq.). «Il est des loix, à l'égard des gouvernemens, comme des remèdes à l'égard des maladies. [...] Si les gouvernemens étoient bien organisés, ils n'auroient pas besoin de tant de loix » (Cat. 69). 
Il en est ainsi aujourd'hui. Il en fut ainsi de tout temps et partout. Boissel ne croit pas à l' «âge d'or » chanté et décrit par les poètes. Il n'y voit qu'une fable des poètes antiques qui l'ont évoqué sans nous nommer les instruments de sa mise en œuvre (Cat. 109). Le siècle d'or ne serait pas derrière nous, mais devant nous.

Telle est la critique de Boissel. Et sa conclusion! Tout ce qui existe ne mérite que de périr! La propriété privée, le mariage (la famille), les religions doivent être entièrement supprimés. "Comment concilier ces institutions avec les droits inaliénables \& imprescriptibles des hommes? Étouffons donc cet ordre, ce culte \& ces institutions désastreux » (Cat. 71).

Et ensuite, alors? Doit-on, comme le prêche Rousseau, détruire la civilisation et retourner à l'état de nature? Non, répond notre auteur. La théorie de Rousseau du caractère pernicieux de la civilisation et de la promiscuité des hommes dans des communautés ordonnées, sa négation de la première et sa sommation à l'abroger, reposent simplement sur un malentendu. Jean-Jacques est parvenu à cette conclusion erronée parce qu'il a considéré la propriété privée non pas seulement comme la base de notre ordre social, mais comme la base de tout ordre social. L'ordre de la propriété privée lui apparaît comme l'ordre social $\kappa \dot{\alpha} \tau \tau^{\prime} \dot{\varepsilon} \xi o x \eta^{\prime} \nu$ [par excellence. NdT]. Et comme il reconnaît pleinement les maux inévitables et terribles de la propriété privée, tout en considérant impossible un ordre social sans celle ci, il en vient donc naturellement à le refuser. En acceptant les prémisses de Rousseau, on ne peut pas se prémunir contre ses conclusions. Il n'y a donc aucune autre issue que celle qu'il recommande. Mais les hypothèses de Rousseau sont fausses. La société établie sur la base de la propriété privée n'est pas la société. Elle en est tout au plus une forme. Et encore! Car il n'existe en réalité qu'un seul ordre social qui mérite ce nom, celui qui se donne pour principe non l'égoïsme mais l'altruisme. Cet ordre social modèle doit d'abord être fondé. «L'auteur du discours sur l'origine [...] de l'inégalité parmi les hommes s'est trompé quand il a dit $[\ldots]$ que celui qui le premier s'était arrogé le droit de propriété devait être regardé comme le vrai fondateur de la société civile. Ce n'était pas le vrai, mais le faux fondateur, le premier usurpateur » (Cat. 63). Si Rousseau avait réfléchi plus mûrement, il aurait naturellement fini lui aussi par être convaincu du caractère pernicieux de l'ordre actuel des choses. Il aurait cependant également reconnu, d'autre part, la nécessité d'une promiscuité sociale des hommes ordonnée, reposant sur des bases véritablement raisonnables, ainsi que les multiples avantages qu'elle offrirait (Cat. 64-65, ainsi que Les Entretiens du Père Gérard, passim). 
L'abandon de l'ordre social ne peut en aucune façon nous aider, pense Boissel, pas plus que les palliatifs qui sont maintes fois réclamés, par exemple la loi agraire. " La loi agraire, ou le partage égal des terres, ne pourra avoir lieu, comme ne pouvant déraciner la cause du mal » (Les Entretiens, 175-176). L'aide ne viendra que de la reconstruction de l'ordre social sur une base altruiste.

Nous avons vu dans l'introduction à la présentation de son système ce que Boissel entend par là. Son programme négatif se déduit alors de lui-même. Il culmine par la proposition de détruire toutes les institutions à caractère individualiste et celles qui en résultent. C'est-à-dire tous les apanages individuels et corporatistes; puis aussi les droits individuels conduisant à la domination, et le plus important d'entre eux, le droit de propriété; la famille et son fondement, le mariage; enfin, les religions et toutes les institutions religieuses (Cat. $60 \mathrm{sq}$., $178 \mathrm{sq}$., $194 \mathrm{sq}$. et passim, ainsi que Les Entretiens, passim).

Pour ce qui concerne d'abord la religion, la tolérance absolue doit dorénavant régner. Chacun pourrait adopter la vénération de Dieu qui lui convient. La libre expression d'idée serait même concédée aux athées et aux matérialistes - même s'ils ne pourraient à vrai dire être perçus que comme des insensés. Bien évidemment, à l'avenir, il conviendrait d'établir très rigoureusement la séparation de l'Église et de l'État, de bannir tout enseignement religieux des écoles et des organismes publics. Les prêtres, s'ils voulaient camper sur leurs positions, ne devraient pas être dérangés dans leur vie privée, mais se soumettre pour le reste aux mêmes devoirs que les autres membres de la société (cf. Cat. 185-186, 194 sq., Les Entretiens 17 sq. et passim).

Il conviendrait de même de supprimer complètement le mariage. À sa place devrait être introduit l'amour libre conforme à la nature. Dorénavant, « on n'établiroit de règle, pour l'union de l'homme et de la femme, que celles qui ne pourroient tendre qu'à remplir les vues de la nature [...], qu'à bonifier, qu'à augmenter la population, ou même pour en arrêter le trop grand progrès, en cas de besoin » $(\text { Cat. } 79)^{14}$.

À l'avenir, il n'y aurait que des pères et des mères, des frères et des sœurs (Cat. 177 sq., Les Entretiens 177-178). Les enfants issus d'une union libre entre un homme et une femme seraient élevés par la société; la paternité et la maternité dans le sens actuel, ainsi que la famille, disparaîtraient; elles revendiqueraient l'amour et l'obéissance de tous les

(14) Notre auteur ne dit cependant pas comment. Ce passage démontre cependant que le courant d'idée que l'on nomme malthusianisme ne lui était pas complètement étranger. 
enfants. Ceux-ci en retour devraient aimer et honorer également tous les pères et mères, et leur obéir à égale mesure.

Naturellement, toute propriété privée serait également proscrite dans la société idéale à venir. Tous les biens appartiendraient à la communauté et seraient répartis entre ses membres en fonction des besoins de chacun. L'ordre nouveau reposerait sur l'égalité et l'égalité de droit à l'existence de tous les membres de la société.

Il ne pourrait naturellement pas être question d'accepter le droit au plein rendement du travail, car celui-ci est l'exacte contradiction de l'accomplissement du droit à l'existence et que les personnes dans l'incapacité de travailler - enfants, malades, invalides, vieillards - devraient être maintenues en dehors du rendement du travail global.

Boissel trouve cela d'autant moins injuste que le besoin, et non l'ampleur et le degré du travail, soit pris pour critère de répartition des biens. Imprégnés de ce principe qu'ils seraient là pour travailler pour leurs semblables et pour trouver leur bonheur dans celui de la communauté, les membres de la future société ne percevraient aucune injustice dans ce mode de répartition. "Le citoyen », estime notre auteur, auquel la nature aurait conféré « plus de force ou plus d'intelligence, seroit destiné à remplir les fonctions qui demanderoient plus de lumière ou de force, sans qu'il lui fût permis de s'en prévaloir vis-à-vis de ses semblables, dont le cœur lui tiendront bon compte de ses talens \& de son zèle » (Cat. 178).

Comme, dans la nouvelle société, toutes les personnes capables de travailler devraient travailler et que l'existence de chacun serait garantie par le rendement du travail de la société, il ne pourrait y avoir aucune personne sans travail et la question du droit au travail s'annulerait d'ellemême.

Mentionnons encore qu'il n'y aurait dans le nouvel État ni argent ni impôt au sens où nous l'entendons aujourd'hui, c'est-à-dire « l'obligation de contribuer aux frais du maintien $\&$ de l'exécution de notre ordre social et juridique avilissant ». Dans l'ordre idéal futur, chacun mettrait sa personne et toutes ses compétences au service de la communauté (Cat. 148-149).

Boissel ne donne pas d'autres détails sur sa société modèle. Ceci peut d'ailleurs se comprendre facilement : d'une part, il n'est pas question pour lui, a priori, d'écrire une utopie dans le sens habituel du terme; d'autre part, il est profondément convaincu que, si la vérité et la raison venaient à l'emporter, tout s'imbriquerait au mieux et rendrait les conflits d'intérêt entre les individus et les groupes tout bonnement impensables. 
Boissel n'explique guère qui devrait prendre la direction de la nouvelle société et de quelle manière celle-ci devrait évoluer. L'éducation devrait, selon lui, être en tout cas remise entre les mains des vieux et des sages - qui n'auraient désormais aucun intérêt contraire au bien-être général (Cat. 11).

Nul n'est besoin de souligner particulièrement que notre auteur attend de la mise en œuvre de ses propositions un résultat des plus brillants, le royaume des cieux sur terre. Seules la vertu, la sagesse et la morale animeraient alors les hommes. "S'il naissoit quelque monstre perturbateur, $\&$ de la sûreté \& de l'ordre publics, il seroit dépouillé de son uniforme \& même de sa liberté; si c'étoit des monstres du dehors, après avoir épuisé toutes les ressources de la raison $\&$ de la douceur, pour les humaniser, on feroit usage de tous les moyens que l'éducation auroit appris pour les étouffer, si on pouvoit s'en garantir autrement ${ }^{15}$; mais une fois l'ordre moral \& l'éducation sociale établis \& propagés, ces sortes de productions seroient moralement impossibles ou impuissantes » (Cat. 187). [...]

La notion de propriété privée ne doit provisoirement pas être modifiée durant le stade de transition. Seulement, comme durant celui-ci le droit de tous à l'existence serait garanti, cependant pas à la même existence, cette concession ne signifie au fond pas grand chose. Du revenu total de la nation devrait en effet être déduit une part de biens - sous la forme d'un impôt infligé aux possédants - qui serait nécessaire à l'éducation et au maintien des personnes dans l'incapacité, pour une quelconque raison, de travailler. Les propriétaires ne pourraient disposer que du surplus.

Ceci aurait bien évidemment diverses conséquences, qui, tout en laissant inchangée la notion de propriété privée, la taxeraient de manière si forte que sa valeur en deviendrait effectivement illusoire pour le propriétaire.

Boissel n'a naturellement pas peur non plus d'en tirer ces conclusions. Comme les produits agricoles et industriels de base sont d'une grande importance pour le maintien de la communauté, on ne pourrait pas - pense-t-il - laisser les propriétaires décider s'ils produisent, et comment. Ils seraient alors à considérer comme de simples « dépositaires comptables, garans et responsables » de l'État. Ce raisonnement amène naturellement Boissel à exiger : 1) que soient punies la négligence de la culture du sol et toute décision au détriment du public avec la rétrocession

(15) Boissel se montre dans le Catéchisme du genre humain un opposant inconditionnel à la peine de mort. Cf. p. 107-108. 
$\mathrm{du}$ sol et des biens fonciers ainsi que de ses produits aux municipalités concernées; 2) que soient construits dans les différentes communes des entrepôts publics où devraient être déposés tous les produits agricoles contre paiement de l'État.

Personne ne pourra revendiquer le droit à l'existence, à moins qu'il ne l'obtienne par son travail, à la seule exception des personnes dans l'incapacité de travailler (enfants, vieillards, malades et invalides). Des asiles spéciaux devront être construits dans toutes les communes pour l'entretien de ces trois dernières catégories.

Comme, pendant ce stade de transition, le contrat de travail libre serait encore appliqué, Boissel se voit naturellement contraint de reconnaître le droit au travail comme le complément logique du droit à l'existence. Il sollicite ainsi la construction d'ateliers publics dans chaque commune, où les personnes sans travail trouveraient de l'emploi.

Durant la période de transition, le mariage doit adopter le caractère d'un simple contrat de droit privé, le divorce libre, être autorisé sans condition, et l'intervention de l'État à cette occasion, se limiter à prendre une décision lorsque les revendications relevant du droit du patrimoine font l'objet d'un litige entre les époux.

Comme la séparation sans condition de l'Église d'une part et de l'État et de la société d'autre part en résulterait immédiatement, et que la religion deviendrait une affaire privée au sens le plus strict du terme, les églises, les cloîtres et leurs possessions, devenus libres, pourraient être utilisés à des fins scolaires.

Naturellement, dans le nouvel État, les guerres offensives cesseraient immédiatement; l'État ne se défendrait qu'en cas d'attaque. Entre temps, l'« éducation sociale » veillerait à ce que la nouvelle génération conçoive le dégoût le plus complet de la propriété privée et de l'ordre juridique fondé sur cette dernière, et qu'elle soit inversement imprégnée des principes de l'amour du prochain le plus noble et le plus pur.

Concernant l'éducation, celle-ci serait distincte pour les différents sexes. L'enseignement devrait naturellement - comme à l'avenir - être le même pour les deux sexes et pour tous les individus pour la théorie et les principes; mais pour le reste il faudrait tenir compte des différentes dispositions mentales et physiques. La décision en ce sens reviendrait aux « mères les plus tendres, les plus sages, les plus éclairées et les plus expérimentées » (Cat. 182).

Aux écoles devraient être associés des ateliers et des établissements de formation professionnelle dans les domaines de l'agriculture, de l'industrie et de l'art. 
Mentionnons encore que Boissel veut tout particulièrement honorer le sexe féminin, en raison de ses nombreuses qualités - comme mères, nourrices et éducatrices de l'humanité, et en raison du bonheur que leur amour confère à l'homme - en les élevant dans des «temples magnifiques » (Cat. 180).

Les frais de tous ces établissements seraient naturellement pris en charge par les possédants. Ils ne seraient cependant, d'après Boissel, que justes et peu élevés, car ils ne donneraient droit en soi à rien de plus qu'à la satisfaction «frugale » des besoins, et cela encore seulement à raison du travail effectué. Il s'étonnerait tout aussi peu si la taxation élevée et la responsabilité vis-à-vis de l'État liée à la propriété suscitaient en peu de temps chez les propriétaires privés une aussi grande horreur de leurs droits privés, que celle inculquée à leurs enfants par l'« éducation sociale». Car voilà bien le but dont il rêve. S'il était atteint, plus rien ne s'opposerait à l'avènement du royaume de la vertu et de la vérité, qui pourrait de ce fait se réaliser d'autant plus rapidement.

J'en ai terminé. Il n'est pas dans mon intention de critiquer le système de Boissel; et encore moins de m'y opposer ou de le contredire sérieusement; ce qui serait d'ailleurs déplacé, en raison de ses nombreuses absurdités. Les exagérations et conclusions parfois vraiment déraisonnables de Boissel - aux côtés des nombreuses idées justes et recommandables - se dénoncent d'elles-mêmes. [...]

Je n'avais donc dès le début pour intention que de sortir de l'oubli et d'esquisser brièvement les idées d'un homme qui n'a certes personnellement joué aucun rôle prédominant, mais dont les écrits semblent avoir été beaucoup lus et avoir ainsi stimulé les futurs co-conjurés de Babeuf à participer à l'entreprise de ce dernier. Que Boissel n'apparaisse pas luimême dans cette conjuration et n'y ait aucunement pris part peut s'expliquer par le fait qu'il avait des pensées audacieuses, mais qu'il ne l'était précisément pas. Il avait en outre toujours prêché dans le Catéchisme la régénération de l'humanité par des réformes pacifiques. [...]

Quoi qu'il en soit : cela n'enlève naturellement rien au fait que Boissel doit être considéré comme l'un des précurseurs les plus audacieux et les plus cohérents du socialisme moderne, et qu'il est autorisé à revendiquer une place significative dans la genèse de ce dernier. L'exigence du droit à l'existence et - comme succédané de celui-ci au sein de notre ordre de droit privé - du droit au travail ; l'idée selon laquelle tous les phénomènes culturels - scientifiques, religieux, politiques - dépendent des rapports de force économiques; l'idée, également tout récemment souli- 
gnée avec force par des juristes érudits ${ }^{16}$, selon laquelle l'ordre juridique dans son ensemble a été créé par les classes possédantes en tenant exclusivement compte de leurs intérêts et au service de ces derniers ; la revendication d'un enseignement obligatoire général; l'enseignement égal, public et gratuit et l'entretien des élèves durant la période d'enseignement sur les deniers publics; l'émancipation des femmes et l'égalité complète des deux sexes; le libre divorce; la paix perpétuelle et la suppression des guerres, etc. Tout cela se trouve dans les écrits de Boissel, en marge de sa critique du caractère pernicieux de la propriété privée.

Une chose manque : la critique du mode de production capitaliste. Mais rien n'est plus normal. Dans la France d'alors, la propriété foncière était encore le plus important facteur de puissance économique. Tout le pouvoir politique y était encore concentré. Le capital mobile - aussi puissante qu'ait déjà réellement été son influence -, n'avait pas encore imposé la reconnaissance juridique de cette position de force. Ceci ne vint qu'avec la Révolution. Le développement de la production capitaliste n'est de même qu'une conséquence de la victoire totale du libéralisme économique, entraînée par la Révolution. C'est pourquoi Boissel considère et lutte en premier lieu contre la propriété privée du sol et des biens fonciers. Nous avons cependant vu qu'il n'en reste pas là. Les écoles socialistes postérieures reprendront et poursuivront sa critique là où il avait dû l'arrêter.

Nous devons donc voir en Boissel un penseur qui représente la dernière phase d'évolution de l'ancien socialisme utopique au socialisme moderne.

(16) Cf. en particulier Anton Menger, Das bürgerliche Recht und die besitzlosen Volksklassen [Le droit bourgeois et les classes populaires non-possédantes], dans Braun, Archiv für soziale Gesetzgebung und Statistik [Archives de législation sociale et de statistique], vol. II et III, 1889 et 1890 ; également en tiré à part, $1^{\mathrm{e}}$ et $2^{\mathrm{e}}$ éd., Laupp, Tübingen, 1890. 